Copyright 2017 Acoustical Society of America. This article may be downloaded for personal use only. Any other use requires prior permission of the author and the Acoustical Society of America.

The following article appeared in (Journal of the Acouctical Society of America,142(2), pp.890-901) and may be found at

http://scitation.aip.org/content/asa/journal/jasa/142/2/10.1121/1.4995999?a email=author 


\title{
Dynamic mass density of resonant metamaterials with homogeneous inclusions
}

\author{
Guy Bonnet ${ }^{a)}$ and Vincent Monchiet \\ Université Paris-Est, Laboratoire Modélisation et Simulation Multi-Echelle, MSME UMR 8208 CNRS, \\ 5 boulevard Descartes, 77454 Marne la Vallée, France
}

(Received 29 October 2016; revised 18 June 2017; accepted 3 July 2017; published online 14 August 2017)

\begin{abstract}
The occurrence of a negative dynamic mass density is a striking property of metamaterials. It appears when an inner local resonance is present. Results coming from an asymptotic theory are recalled briefly, showing the scaling of physical properties leading to inner resonance in elastic composites containing homogeneous soft inclusions, with negligible scattering of waves traveling through the matrix. This appears for a large contrast of elastic properties between matrix and inclusion. The frequency-dependent dynamic mass density depends on the resonance frequencies of the inner inclusions and on their related participation factors. Having solved the dynamic elasticity problem, these physical quantities are provided in the case of homogeneous cylindrical and spherical inclusions. It is shown that numerous resonance frequencies do not contribute to the dynamic mass density or have small participation factors, which simplifies significantly the physics involved in the concerned inner resonance phenomena. Finally, non-dimensional resonance frequencies and participation factors are given for both cases of inclusions as functions of the Poisson's ratio, defining completely the dynamic mass density. (C) 2017 Acoustical Society of America.
\end{abstract}

[http://dx.doi.org/10.1121/1.4995999]

$[\mathrm{MRH}]$

Pages: 890-901

\section{INTRODUCTION}

Even if the existence of metamaterials has been predicted initially in the case of electromagnetic properties, ${ }^{1,2}$ acoustic metamaterials have been studied extensively during the last few decades, especially since the production of experiments showing bandgaps when studying the propagation of elastic waves in phononic crystals ${ }^{3}$ followed a few years after by experiments of wave propagation through acoustic metamaterials. ${ }^{4}$ Acoustic metamaterials can have more or less complex internal structures as proven in the numerous papers in this field either studying properties of metamaterials from a theoretical approach ${ }^{5-9}$ and/or showing experimentally the properties of these materials. ${ }^{4,10-14}$ These materials can have "effective" negative dynamic density and/or elastic moduli for some frequency ranges, leading to forbidden frequency bands. A recent review paper on acoustic metamaterials ${ }^{15}$ draws a large picture of this domain, showing that the interesting properties of metamaterials are enhanced by inserting local resonators. These resonators can be a more or less complex combination of plates, beams, spring-mass systems, Helmholtz resonators, or (homogeneous or heterogeneous) inclusions of different shapes. This review paper also reports on several recent studies on the conception of materials having simultaneously negative effective mass density and elastic moduli..$^{8,16,17}$

In the following, the study will be restricted to elastic composites containing inclusions of different shapes. Even so, the amount of literature devoted to this field is impressive. It is known for a long time that every periodic composite

${ }^{\text {a)} E l e c t r o n i c ~ m a i l: ~ g u y . b o n n e t @ u n i v-p a r i s-e s t . f r ~}$ containing inclusions is characterized by forbidden frequency bands, its response to wave propagation being studied usually by Bloch waves. However, these forbidden frequencies appear at high frequencies when the wavelength is on the order of the inclusion size, i.e., the material being considered as a "phononic crystal." A few decades ago, it was recognized that the frequency range of forbidden frequency bands is lowered by introducing inner resonators and that the most simple way to induce inner resonance is to use matrix-inclusion composites containing rigid inclusions surrounded by a soft coating (i.e., "composite inclusions"), the whole being immersed within an elastic matrix. This led to the concept of acoustic metamaterials. The composite inclusions act as "spring-mass" resonators, the mass being provided by the inner inclusion and the spring by the surrounding coating. In the case of a large contrast between elastic properties of coating and matrix, the low resonance frequency of the spring-mass resonator can be reached practically without scattering of the waves by the inclusions. It is the extreme case of "inner-resonance with negligible scattering." Obviously, when the elastic properties of the coating become of the same order as the ones of the matrix, it is necessary to take into account the coupling of the inner resonance with the scattering of waves by the inclusions. Several methods were used to account for this coupling, ${ }^{18-23}$ the most currently used being the Coherent Potential Approximation. ${ }^{24}$ However, in the case of a soft coating, the case of inner resonance with negligible scattering is certainly an interesting comparison model, which becomes fully valid for large contrasts of elastic properties of constituents.

Less work was devoted to homogeneous inclusions, and these works deal mainly with scattering by inner inclusions. However, early works using homogenization theory based 
on asymptotic expansion ${ }^{25,26}$ have shown that inner resonance with negligible scattering can also occur in composites containing homogeneous inclusions. This is the domain of application of the present paper.

The physics of wave propagation through composite materials is complex and a strong physical insight can be gained by using an asymptotic series expansion ${ }^{27}$ of the dynamic solution for different assumptions of the ratios between physical parameters: elastic coefficients and mass densities. First, homogenization results showing the occurrence of bandgaps in periodic elastic composites with inner resonance with negligible scattering were obtained in early works, ${ }^{25,26}$ but with the development of the ideas on metamaterials, new results were obtained with the use of asymptotic expansions in dynamic elasticity. ${ }^{28-38}$ More specifically, Auriault and Boutin $^{28}$ have recently extended the method of asymptotic expansion to materials containing composite inclusions and characterized by inner resonance with negligible scattering. It is worthwhile noticing that, compared with results coming from other publications in this field, an important aspect does appear in the papers written by Auriault and co-workers that are at the basis of this paper: ${ }^{25,26,28}$ it is shown indeed in these papers that the use of different scalings on physical properties can lead to different macroscopic behaviours. In the case of a strong contrast, the method proves the occurrence of inner resonance with negligible scattering; it provides the structure of the effective constitutive equations and the local elasticity problems (usually called "localization problems" or "cell problems") to be solved in order to obtain the macroscopic physical properties, more particularly the dynamic mass density. It is worth noticing that in the absence of strong contrasts, the predicted dynamic behaviour can be non-local in space $^{39-42}$ while the behaviour described in the case of inner resonance with negligible scattering is local in space (while non-local in time). This point is of importance for modeling wave propagation through a structure which would be made of such a metamaterial.

In a recent paper ${ }^{43}$ the dynamic mass density has been obtained by solving the localization problem described by the homogenization theory in the case of materials displaying inner resonance with negligible scattering and containing spherical or cylindrical composite inclusions that act as springmass resonators. Under suitable conditions, such a dynamic mass density can be obtained by studying the static behaviour (spring effect) of the composite inclusion. By comparison, in the case of homogeneous inclusions described thereafter, the dynamic mass density involves the full dynamic behaviour of the inclusions.

In Sec. II, the results of asymptotic expansions in the case of materials containing homogeneous inclusions are synthesized and discussed, showing that suitable ratios of the physical parameters can lead to inner resonance with negligible scattering. The method also specifies the dynamic boundary value problem ("cell problem") to be solved in order to provide the dynamic mass density. In Sec. III, the inner motion of inclusions made of cylindrical fibers is studied: the eigenfrequencies are obtained and then participation factors and dynamic mass densities of these resonators are given in a closed form. The case of spherical inclusions is studied in Sec. IV and the full solution of the local elasticity problem is given in this case, providing again the eigenfrequencies, participation factors, and dynamic mass densities. Finally, numerical applications are presented in Sec. V.

\section{DYNAMIC BEHAVIOUR OF ELASTIC COMPOSITES CONTAINING HOMOGENEOUS INCLUSIONS}

This section recalls briefly the main results from homogenization using asymptotic theory ${ }^{25,26,28}$ and the underlying assumptions that justify the method used in Secs. II A-IID to obtain the dynamic mass density. Let us consider a periodic elastic composite material made up of a matrix containing homogeneous inclusions. This composite material is defined by the geometry of the periodic cell containing the inclusions and by the physical properties of the constituent materials. These constituents are assumed elastic and isotropic. They are characterized by their mass densities $\rho^{(s)}$, their volume concentrations $c^{(s)}$, and their Lamé elastic parameters $\lambda^{(s)}, \mu^{(s)}$. (s) corresponds to the matrix $(m)$ or the inclusion (i). The effective behaviour of the composite depends strongly on the scaling parameters. In a first step, these scaling parameters are defined and, next, the scaling assumptions leading to the occurrence of a negative mass density will be provided.

\section{A. Scaling parameters}

A first parameter is the geometric scaling ratio defined by $\epsilon=l / L$, where $l$ is the size of the periodic cell and $L$ is the order of magnitude of the wavelength within the matrix in a chosen frequency range. The case of interest is when the size of the periodic cell is small compared with $L$ and therefore $\epsilon \ll 1$.

Other scaling parameters contain the various physical parameters that characterize the constituents of the composite.

They comprise:

(i) the ratio between the mass densities of the constituents $\rho^{(i)} / \rho^{(m)}$ and

(ii) the ratio between the orders of magnitude of the elastic coefficients of the different constituents.

The order of magnitude of the elasticity coefficients of a given material will be defined by the value of $a^{(s)}=\lambda^{(s)}$ $+2 \mu^{(s)}$ (i.e., a norm of the elasticity tensor). The scaling ratio related to elastic coefficients will be defined by $\left(\lambda^{(i)}+2 \mu^{(i)}\right) /$ $\left(\lambda^{(m)}+2 \mu^{(m)}\right)=a^{(i)} / a^{(m)}$.

\section{B. Scaling assumptions leading to the occurrence of a negative dynamic mass density}

Using asymptotic expansion along the scaling parameter $\varepsilon$, Auriault and Bonnet ${ }^{25}$ (see also Refs. 26 and 28) studied the effect of different values of the previously defined scaling ratios on the overall behaviour of the composite. These authors showed that the resonance of the inner inclusions can be obtained when the inclusions are very soft compared with the matrix, the densities being of the same order of magnitude. More precisely, the inner resonance is obtained 
when the scaling ratios meet the following conditions: $a^{(i)} / a^{(m)}=O\left(\epsilon^{2}\right)$ for the elastic parameters, and $\rho^{(i)} / \rho^{(m)}=0(1)$ for the mass densities.

\section{The dynamic mass density}

Under the previously defined conditions, the composite has an overall effective behaviour for harmonic time excitation at radial frequency $\omega$ that is characterized by the dynamic equation

$$
\frac{\partial\left(a_{i j k l}^{(\text {eff })} \varepsilon_{k l}\right)}{\partial x_{j}}+\omega^{2} \rho_{i j}^{(\text {eff })} u_{j}=0,
$$

where $a_{i j k l}^{\text {(eff) }}$ are the effective elastic coefficients of the composite, $\varepsilon_{k l}$ are the components of the overall strain tensor, $u_{j}$ are the components of the displacement, and $\rho_{i j}^{\text {(eff) }}$ are the components of a frequency-dependent dynamic mass density. With a periodic array of inclusions, the effective elastic behaviour is not isotropic, contrary to the behaviour of the constituents. Due to the scaling assumptions, the inclusions are very soft compared with the matrix. As a consequence, the effective elastic coefficients $a_{i j k l}^{(\text {eff })}$ can be computed as for a matrix that contains voids in place of inclusions by usual means to obtain effective properties of heterogeneous elastic materials.

Concerning the components $\rho_{i j}^{\text {(eff) }}$ of the mass density, they must be considered as the ones of a tensor of second rank. In the following, it will be assumed that the inclusions have three orthogonal symmetry planes. In this case, the dynamic mass density is diagonal for a coordinate system whose axes are parallel to the symmetry planes, with

$$
\rho_{i i}^{(\mathrm{eff})}(\omega)=\langle\rho\rangle+c^{(i)} \rho^{(i)} h_{i i}(\omega),
$$

where Einstein's convention summation must not be applied to the repeated index $i$ in $\rho_{i i}^{\text {(eff) }}, h_{i i}$. $\langle\rho\rangle$ is the volume average of the density within the periodic cell and $c^{(i)}$ is the volume concentration of inclusions. $h_{i i}$ are the components of a diagonal second order tensor.

The homogenization method provides the localization problems (cell problems) allowing to compute the effective elastic properties and the tensor of components $h_{i j}$, as recalled in Appendix A. The asymptotic process proves that, at the first order, due to the large wavelength within the matrix, the fluctuation of displacement of the matrix $\vec{u}^{(m)}$ within the periodic cell is on the order of $\varepsilon$ (while the fluctuations of strain field are of the same order as the macroscopic strain). It means that the displacement and acceleration of the matrix are nearly constant inside a periodic cell. As a consequence, the localization problem obtained by homogenization theory recalled in Appendix A corresponds to a dynamic localization problem within the inclusion moved by a uniform acceleration induced by the matrix. It can be expressed by using the relative displacement $\vec{w}=\vec{u}^{(i)}-\vec{u}^{(m)}$. This relative displacement is null over the boundary of the inclusion and is the solution of

$$
\begin{aligned}
& \mu^{(i)} \Delta \vec{w}+\left(\lambda^{(i)}+\mu^{(i)}\right) \operatorname{grad}(\operatorname{div}(\vec{w}))+\rho^{(i)}(\omega)^{2} \vec{w} \\
& \quad=-\rho^{(i)}(\omega)^{2} \vec{u}^{(m)} .
\end{aligned}
$$

It means that the motion within a given inclusion, in a local reference frame moving with the matrix, is impulsed by the inertial acceleration $-\rho^{(i)}(\omega)^{2} \vec{u}^{(m)}$.

The solution of this problem can be expressed by using the eigenfrequencies $\omega^{p}$ and eigenmodes $\vec{u}^{p}$ of the inclusions for fixed boundaries in the reference frame moving with the matrix, i.e., the solutions of

$$
\mu^{(i)} \Delta \vec{u}^{p}+\left(\lambda^{(i)}+\mu^{(i)}\right) \operatorname{grad}\left(\operatorname{div}\left(\vec{u}^{p}\right)\right)+\rho^{(i)}\left(\omega^{p}\right)^{2} \vec{u}^{p}=0,
$$

with $\vec{u}^{p}=0$ on the boundary of the inclusion.

Finally, for an acceleration of the matrix given by $\gamma \vec{e}_{j}$ where $\vec{e}_{j}$ is a unit vector along one of the axes, the related component $h_{j j}(\omega)$ is given by

$$
h_{j j}(\omega)=\sum_{p=1}^{\infty} K_{j j}^{p} \cdot \frac{1}{\left(\frac{\omega^{p}}{\omega}\right)^{2}-1},
$$

where the participation factors $K_{j j}^{p}$ do not depend on $\omega$ and are given by

$$
K_{j j}^{p}=\frac{\left(\left\langle\vec{u}^{p}\right\rangle^{(i)} \cdot \vec{e}_{j}\right)^{2}}{\left\langle\left\|\vec{u}^{p}\right\|^{2}\right\rangle^{(i)}},
$$

the volume average $\langle f\rangle^{(i)}$ being computed over the volume of the inclusion. Due to the dependence on $\omega, h_{j j}$ tends to minus infinity just above each resonance frequency. As a consequence, the related component of the overall dynamic mass density $\rho_{j j}^{\text {(eff) }}$ tends also to minus infinity and remains negative until its negative frequency dependent part is equilibrated by the static overall mass density $\langle\rho\rangle$ in Eq. (2).

It is worthwhile noticing that, due to the condition at the boundary of the inclusion (induced obviously by the assumption of "negligible scattering"), the contributions of different separate inclusions within a periodic cell are completely independent and can be summed in order to compute the overall dynamic mass density for any distribution of inclusions.

Finally, the eigenfrequencies and eigenmodes $\left(\omega^{p}, \vec{u}^{p}\right)$ must be obtained for each direction of $\vec{e}_{j}$ by solving a "localized eigenmodes problem" [Eq. (4)]. Next, the averages in Eq. (6) are computed to produce the three components of the participation factors. In Secs. III and IV, the localization problems will be solved for cylindrical and spherical inclusions.

\section{Physical discussion of the results}

The results obtained from asymptotic expansion may be surprising by some aspects. Indeed, these results show that the inclusion behaves as if the surrounding material moves uniformly at its boundary. In addition, the field inside the 
inclusion does not depend on the wavelength within the surrounding material and is therefore not affected by the scattering of these waves by the inclusions.

Obviously, the displacement within the matrix is not exactly uniform due to the varying strain around the inclusion. However, the ratio of strain fields between matrix and inclusion is very large, due to the elasticity contrast, and as a consequence, the fluctuation of displacement around the inclusion is very small compared with the displacement inside the inclusion and may be considered as negligible. The absence of scattering comes naturally from the initial assumption of a large ratio between the wavelength related to the propagation within the matrix and the size of inclusions. Finally, the results coming from the asymptotic theory appear as physically sound. Indeed, numerous devices entering the composition of metamaterials use inner resonators whose resonance appears at low frequencies, below frequencies inducing a scattering by the cells containing the resonators (Helmholtz resonators, spring-mass resonators, etc.).

Another point of discussion is the fact that the dynamic density tends to infinity at the resonance frequencies. Obviously, this result comes from the assumption of perfectly elastic materials. For real materials, the physical damping must be taken into account. This can be affected by assuming the inclusion material as viscoelastic.

In this case, the viscoelastic behaviour of the inclusion material can be taken into account by using complex and frequency dependent elastic moduli. As a result of the use of complex moduli, the orthogonality of modes is no more ensured. Due to this loss of orthogonality, the method presented previously cannot be used as such in the case of viscoelasticity and will not be studied thereafter. For a slight damping, an asymptotic procedure can again be used in order to provide the viscoelastic solution. ${ }^{28}$ It leads to a finite value of the dynamic density and to a small shift of the resonance frequency.

\section{DYNAMIC MASS DENSITY FOR CYLINDRICAL INCLUSIONS}

Let us consider a composite containing long parallel cylindrical inclusions, i.e., long cylindrical fibers of radius $a$. As seen in Sec. II, the dynamic contribution to the dynamic mass density depends only on the properties of the inclusions. Therefore, in the following, the notation $\lambda^{(i)}$ $=\lambda, \mu^{(i)}=\mu, \rho^{(i)}=\rho$ will be used. In a first step, the general dynamic displacement within the fiber is given by using separate variables. Next, it will be shown that numerous contributions to this general field do not provide contributions to the participation factors. Finally, the expression of the dynamic mass density will be given.

\section{A. Dynamic displacement field within a cylindrical domain}

Different solutions exist for the dynamic displacement field within a cylindrical domain ${ }^{44,45}$ using separate variables. The expression below is given in Eringen and Suhubi ${ }^{44}$ [Eqs. (8.9.17) and (8.9.18)] where it is shown that the displacement field is the superposition for integer values of $n$ of the following partial displacements:

$$
\begin{aligned}
u_{r}= & \frac{1}{r}\left[A_{1} U_{1}(\alpha r) \cos n \theta e^{ \pm i \gamma_{p} z}+B_{1} U_{2}(\beta r) \sin n \theta e^{ \pm i \gamma_{q} z}\right. \\
& \left.+C_{1} U_{3}(\beta r) \cos n \theta e^{ \pm i \gamma_{q} z}\right], \\
u_{\theta}= & \frac{1}{r}\left[A_{1} V_{1}(\alpha r) \sin n \theta e^{ \pm i \gamma_{p} z}+B_{1} V_{2}(\beta r) \cos n \theta e^{ \pm i \gamma_{q} z}\right. \\
& \left.+C_{1} V_{3}(\beta r) \sin n \theta e^{ \pm i \gamma_{q} z}\right], \\
u_{z}= & A_{1} W_{1}(\alpha r) \cos n \theta e^{ \pm i \gamma_{p} z}+C_{1} W_{3}(\beta r) \cos n \theta e^{ \pm i \gamma_{q} z}
\end{aligned}
$$

for $n=0, \ldots, \infty$, where $\alpha=\omega / c_{p}, \beta=\omega / c_{s}$ are the wave numbers related to the celerities of compressional and shear waves given by $c_{p}=\sqrt{(\lambda+2 \mu) / \rho}, c_{s}=\sqrt{\mu / \rho}$, with an "alternative expression" obtained by replacing the cos terms by $\sin$ ones (and reversely) and the constants $A_{1}, B_{1}, C_{1}$ by another set of constants $A_{2}, B_{2}, C_{2}$.

$U_{1}, U_{2}, U_{3}, V_{1}, V_{2}, V_{3}, W_{1}, W_{3}$ are functions of $r$ which are expressed by using Bessel functions.

As shown previously, the displacement field is sought for acceleration parallel to one of the base vectors $\vec{e}_{j}$. This displacement field has the symmetry with respect to the planes containing two coordinate axes. As a consequence, the symmetry induces that $\gamma_{p}=\gamma_{q}=0$. From the expression of the radial components, it comes that $U_{3}, V_{3}$, and $W_{1}$ are null. As another consequence of the symmetry, the constants $B_{1}, A_{2}, C_{2}$ are null. Finally, replacing $A_{1}, B_{2}, C_{1}$ by $A, B, C$, it comes that the displacement field is given by

$$
\begin{aligned}
u_{r} & =\frac{1}{r}\left[A U_{1}(\alpha r) \cos (n \theta)+B U_{2}(\beta r) \cos (n \theta)\right] \\
& =u_{r}^{0} \cos (n \theta), \\
u_{\theta} & =\frac{1}{r}\left[A V_{1}(\alpha r) \sin (n \theta)+B V_{2}(\beta r) \sin (n \theta)\right] \\
& =u_{\theta}^{0} \sin (n \theta), \\
u_{z} & =C W_{3}(\beta r) \cos (n \theta) .
\end{aligned}
$$

The radial dependence is described by the following functions:

$$
\begin{aligned}
& U_{1}(\alpha r)=\alpha r J_{n-1}(\alpha r)-n J_{n}(\alpha r), \\
& U_{2}(\beta r)=n J_{n}(\beta r), \\
& V_{1}(\alpha r)=-n J_{n}(\alpha r), \\
& V_{2}(\beta r)=-\beta r J_{n-1}(\beta r)+n J_{n}(\beta r), \\
& W_{3}(\beta r)=\beta^{2} J_{n}(\beta r),
\end{aligned}
$$

where $J_{n}$ is the $n$th order Bessel function of first kind. It can be seen that this displacement field contains two independent fields: a first one with components $\left(u_{r}, u_{\theta}\right)$ and a second one corresponding to $u_{z}$. The first one corresponds to a transversal displacement and the second one to a longitudinal displacement.

At this stage, the expression of the displacement field contains the components related to any value of $n$. However, it will be shown now that only a few of them contribute to the participation factors. 


\section{B. Selection of the modes contributing to the dynamic mass density}

As seen in Sec. II, the participation factors are functions of the average of the projection of the displacement field along the direction of the unit vector $\vec{e}_{j}$. In a first step, this average projection is studied, because it is important to select only the modes which contribute to the dynamic mass density.

\section{Transversal motion}

It is assumed that the acceleration is transversal, i.e., $\vec{e}_{j}$ is perpendicular to the axis of the cylinder. Due to the symmetry, the direction of $\vec{e}_{j}$ can be chosen as $\vec{e}_{x}$ corresponding to $\theta=0$. The average of the projection of the displacement field over the section is given by $I_{t} / S$, where $S=\pi a^{2}$ and $I_{t}$ is the integral of the projection of the displacement field onto $\vec{e}_{x}$ given by $I_{t}=\int \vec{u} \cdot \vec{e}_{x} r d r d \theta$, with $\vec{e}_{x}=\cos \theta \vec{e}_{r}$ $-\sin \theta \vec{e}_{\theta}$ and $\vec{u} \cdot \vec{e}_{x}=u_{r}^{0} \cos n \theta \cos \theta-u_{\theta}^{0} \sin n \theta \sin \theta$. The integral over $\theta$ is null except for $n=1$. It implies that only the contributions corresponding to $n=1$ must be taken into account in the computation of the dynamic mass density. Finally, the transversal displacement field is given by

$$
\begin{aligned}
& u_{r}=\frac{1}{r}\left[A U_{1}(\alpha r) \cos \theta+B U_{2}(\beta r) \cos \theta\right]=u_{r}^{0} \cos \theta, \\
& u_{\theta}=\frac{1}{r}\left[A V_{1}(\alpha r) \sin \theta+B V_{2}(\beta r) \sin \theta\right]=u_{\theta}^{0} \sin \theta,
\end{aligned}
$$

with $\quad u_{r}^{0}=(1 / r)\left[A U_{1}(\alpha r)+B U_{2}(\beta r)\right], \quad$ and $\quad u_{\theta}^{0}=(1 /$ $r)\left[A V_{1}(\alpha r)+B V_{2}(\beta r)\right]$ and

$$
\begin{aligned}
& U_{1}(\alpha r)=\alpha r J_{0}(\alpha r)-J_{1}(\alpha r)=r \frac{d J_{1}(\alpha r)}{d r}, \\
& U_{2}(\beta r)=J_{1}(\beta r), \\
& V_{1}(\alpha r)=-J_{1}(\alpha r), \\
& V_{2}(\beta r)=-\beta r J_{0}(\beta r)+J_{1}(\beta r)=-r \frac{d J_{1}(\beta r)}{d r} .
\end{aligned}
$$

\section{Longitudinal motion}

The longitudinal motion is obtained for $\vec{e}_{j}=\vec{e}_{z}$. In this case, the integral of the projection of the displacement field is given by $I_{l}=\int \vec{u} \cdot \vec{e}_{z} r d r d \theta=\int u_{z} r d r d \theta$. It involves the integral over $\theta$ of $\cos n \theta$ which is null except for $n=0$. The longitudinal motion is therefore given by $u_{z}$ $=C W_{3}(\beta r)=D J_{0}(\beta r)$, where $D$ is an undetermined constant.

\section{Computation of the eigenfrequencies}

\section{Transversal motion}

The eigenfrequencies correspond to the displacement fields which comply with the condition of null displacement over the boundary of the cylinder $(r=a)$ and therefore to the linear system

$$
\begin{aligned}
& A U_{1}(\alpha a)+B U_{2}(\beta a)=0, \\
& A V_{1}(\alpha a)+B V_{2}(\beta a)=0 .
\end{aligned}
$$

This homogeneous system has a solution only when its determinant is null and finally the eigenfrequencies equation is given by $U_{1}(\alpha a) V_{2}(\beta a)-U_{2}(\beta a) V_{1}(\alpha a)=0$. Using the expressions of functions $U_{i}, V_{i}$, this relation can be written by using only the non-dimensional frequency $\omega^{*}=\omega a / c_{s}$ as

$$
\begin{aligned}
& -k_{c} \omega^{*} J_{0}\left(k_{c} \omega^{*}\right) J_{0}\left(\omega^{*}\right)+k_{c} J_{0}\left(k_{c} \omega^{*}\right) J_{1}\left(\omega^{*}\right) \\
& \quad+J_{0}\left(\omega^{*}\right) J_{1}\left(k_{c} \omega^{*}\right)=0,
\end{aligned}
$$

where $k_{c}=c_{s} / c_{p}$ is the ratio between celerities of shear and compressional waves, which depends only on the Poisson's ratio $\nu$ and is given by $k_{c}=\sqrt{(1-2 \nu) / 2(1-\nu)}$.

The solutions of this equation in $\omega^{*}$ are the nondimensional eigenfrequencies $\omega^{p *}(p=1, \ldots, \infty)$ related to the transversal modes. Having computed these eigenfrequencies, the eigenmodes are obtained by noticing that $A$ and $B$ must comply with the homogeneous equations and therefore $B=q^{p} \cdot A$ with $q^{p}=-U_{1}\left(k_{c} \omega^{p *}\right) / U_{2}\left(\omega^{p *}\right)$. These eigenmodes are thus defined up to the multiplying constant $A$.

The expression of the displacement field is no more valid for the case of an incompressible material, which corresponds to $k_{c}=0$. However, the limit of the equation for eigenfrequencies when $k_{c}$ tends to 0 can be obtained and leads to $J_{2}\left(\omega^{*}\right)=0$. Therefore, the eigenfrequencies tend to the zeros of Bessel function $J_{2}$ when $k_{c}$ tends to 0 (and also $\nu$ tends to 0.5 ).

\section{Longitudinal motion}

In this case, the non-dimensional eigenfrequencies are the solutions of $J_{0}\left(\omega^{p *}\right)=0$. The non-dimensional eigenfrequencies $\omega^{p *}$ are therefore the zeros of Bessel function $J_{0}$.

\section{Computation of the participation factors}

The participation factors $K_{j j}^{p}$ are given by averages of the projection of the displacement field and of its quadratic norm. These averages involve the integrals of the projection $I_{j}=\int \vec{u} \cdot \vec{e}_{j} d S$ and of the square of norm of the displacement field $M_{j}=\int\|\vec{u}\|^{2} d S$. Using these quantities, $K_{p}$ is given by $K_{j j}^{p}=I_{j}^{2} /\left(S \cdot M_{j}\right)$, where $S$ is the cross section area of the fiber. The integrals $I_{j}$ and $M_{j}$ are given below in a closed form.

\section{Transversal motion}

The integrals involved in the transversal motion are given by

$$
I_{x}=a A \pi\left[J_{1}\left(k_{c} \omega^{p *}\right)+q^{p} J_{1}\left(\omega^{p *}\right)\right]
$$


and

$$
\begin{aligned}
M_{x}= & \pi A^{2}\left[G\left(k_{c} \omega^{p *}\right)+q^{p 2} G\left(\omega^{p *}\right)\right. \\
& \left.+2 q^{p} J_{1}\left(k_{c} \omega^{p *}\right) J_{1}\left(\omega^{p *}\right)\right],
\end{aligned}
$$

where $G(x)=\frac{1}{2}\left[\left(x^{2}-2\right) J_{1}^{2}(x)+x^{2} J_{0}^{2}(x)\right]$. A further simplification can be effected on the participation factors by taking into account the equation for eigenfrequencies [Eq. (7)]. Finally, the participation factors are given by

$$
K_{x x}^{p}=\frac{2 \alpha^{*} \beta^{*} J_{2}\left(\alpha^{*}\right) J_{2}\left(\beta^{*}\right)}{J_{1}\left(\alpha^{*}\right) J_{1}\left(\beta^{*}\right)\left(\alpha^{* 2}+\beta^{* 2}\right)-\alpha^{*} \beta^{*}\left[J_{1}\left(\beta^{*}\right) \beta^{*} J_{0}\left(\alpha^{*}\right)+J_{1}\left(\alpha^{*}\right) \alpha^{*} J_{0}\left(\beta^{*}\right)\right]},
$$

where $\alpha^{*}=k_{c} \omega^{p *}$ and $\beta^{*}=\omega^{p *}$.

It is noteworthy that, in the case of incompressibility, the participation factors tend to 0 , because their numerators contain $J_{2}\left(\beta^{*}\right)$ which tends to zero with $k_{c}$ as observed previously.

\section{Longitudinal motion}

In the same way, the participation factors for the longitudinal motion are given by

$$
\begin{aligned}
& I_{z}=\frac{2 \pi D a^{2}}{\beta^{2}} J_{1}\left(\omega^{p *}\right), \\
& M_{z}=\pi D^{2} a^{2}\left[J_{0}^{2}\left(\omega^{p *}\right)+J_{1}^{2}\left(\omega^{p *}\right)\right], \\
& K_{z z}^{p}=\frac{4 J_{1}^{2}\left(\omega^{p *}\right)}{\omega^{p * 2}\left[J_{0}^{2}\left(\omega^{p *}\right)+J_{1}^{2}\left(\omega^{p *}\right)\right]} .
\end{aligned}
$$

Taking into account the properties of eigenfrequencies finally leads to $K_{z z}^{p}=4 / \beta^{* 2}$.

\section{E. Synthesis of the results for cylindrical inclusions}

The dynamic mass density [Eq. (2)] for cylindrical inclusions of radius $a$ is characterized by two sets of eigenfrequencies.

(i) The set of eigenfrequencies for transversal motion that are solutions of

$$
\begin{aligned}
& -k_{c} \omega^{*} J_{0}\left(k_{c} \omega^{*}\right) J_{0}\left(\omega^{*}\right)+k_{c} J_{0}\left(k_{c} \omega^{*}\right) J_{1}\left(\omega^{*}\right) \\
& \quad+J_{0}\left(\omega^{*}\right) J_{1}\left(k_{c} \omega^{*}\right)=0,
\end{aligned}
$$

where $\omega^{*}$ is the non-dimensional frequency given by $\omega^{*}=\omega a / c_{s}, k_{c}$ is given by $k_{c}=\sqrt{1-2 \nu / 2(1-\nu)}$, and $J_{0}, J_{1}$ are Bessel functions.

(ii) The set of eigenfrequencies for longitudinal motion, solutions of $J_{0}\left(\omega^{p *}\right)=0$.

The participation factors $K_{x x}^{p}=K_{y y}^{p}, K_{z z}^{p}$ for the transversal motion which enter the expression of the dynamic density through Eqs. (2), (5), and (6) are given

(iii) for transversal motion by

$$
K_{x x}^{p}=\frac{2 k_{c} J_{2}\left(k_{c} \omega^{p *}\right) J_{2}\left(\omega^{p *}\right)}{J_{1}\left(k_{c} \omega^{p *}\right) J_{1}\left(\omega^{p *}\right)\left(1+k_{c}^{2}\right)-k_{c} \omega^{p *}\left[J_{1}\left(\omega^{p *}\right) J_{0}\left(k_{c} \omega^{p *}\right)+k_{c} J_{1}\left(k_{c} \omega^{p *}\right) J_{0}\left(\omega^{p *}\right)\right]},
$$

(iv) for longitudinal motion by $K_{z z}^{p}=4 /\left(\omega^{p *}\right)^{2}$.

\section{DYNAMIC MASS DENSITY FOR SPHERICAL INCLUSIONS}

We consider now a composite containing spherical inclusions having a radius $a$. Similar to Sec. III, it is necessary to compute the eigenmodes related to null displacement at the boundary of the inclusions. Numerous papers dealt with eigenfrequencies in the case of free boundary, but the case of fixed boundary is less frequently seen. This problem was studied earlier by Debye ${ }^{46}$ for the estimation of the phonon contribution to the specific heat of solids. Some eigenfrequencies for the fixed boundary problem were obtained also by Schafbuch and Rizzo. ${ }^{47}$ However, to our knowledge, the computation of the participation factors and of the dynamic mass density has never been produced.

Taking into account the symmetry of the problem, the components of the dynamic mass density are the same for any orientation of the acceleration of the solid. Therefore, the participation factors $K_{i j}^{p}$ are the components of an isotropic tensor with $K_{i j}^{p}=k^{p} \delta_{i j}$, where $\delta_{i j}$ is the Kronecker symbol.

\section{A. Displacement field within a sphere}

The displacement field within a sphere is given in classical books. The solution proposed in Eringen and Suhubi ${ }^{44}$ [Eqs. (8.13.14) and (8.13.15)] for a harmonic motion leads to the following expression of the partial components of the displacement field in spherical coordinates: 


$$
\begin{aligned}
u_{r}= & \frac{1}{r}\left[A U_{1}(\alpha r)+C U_{3}(\beta r)\right] P_{n}^{m}(\cos \theta) \exp i(m \varphi), \\
u_{\theta}= & \frac{1}{r}\left[\left[A V_{1}(\alpha r)+C V_{3}(\beta r)\right]\left[n \cdot \cot \theta \cdot P_{n}^{m}(\cos \theta)-\frac{n+m}{\sin \theta} P_{n-1}^{m}(\cos \theta)\right]\right. \\
& \left.+B V_{2}(\beta r) \frac{i m}{\sin \theta} P_{n}^{m}(\cos \theta)\right] \exp i(m \varphi), \\
u_{\varphi}= & \frac{1}{r}\left[\left[A V_{1}(\alpha r)+C V_{3}(\beta r)\right] \cdot \frac{i m}{\sin \theta} P_{n}^{m}(\cos \theta)\right. \\
& \left.-r B V_{2}(\beta r)\left[n \cdot \cot \theta \cdot P_{n}^{m}(\cos \theta)-\frac{n+m}{\sin \theta} P_{n-1}^{m}(\cos \theta)\right]\right] \exp i(m \varphi),
\end{aligned}
$$

where $A, B$, and $C$ are constants, $P_{n}^{m}$ are associated Legendre polynomials and the radial dependence is given by

$$
\begin{aligned}
& U_{1}(\alpha r)=n j_{n}(\alpha r)-\alpha r j_{n+1}(\alpha r), \\
& U_{3}(\beta r)=n(n+1) j_{n}(\beta r), \\
& V_{1}(\alpha r)=j_{n}(\alpha r), \\
& V_{2}(\beta r)=j_{n}(\beta r), \\
& V_{3}(\beta r)=(n+1) j_{n}(\beta r)-\beta r j_{n+1}(\beta r) .
\end{aligned}
$$

These functions are expressed using spherical Bessel functions $^{48} j_{n}$ given by $j_{n}(k r)=\sqrt{\pi / 2 k r} J_{n+1 / 2}(k r)$. The computation of these spherical Bessel functions can be significantly alleviated, because these functions can be obtained by using trigonometric functions as follows: $j_{1}(z)=\sin z / z^{2}$ $-\cos z / z$, and $j_{2}(z)=\left(3 / z^{3}-1 / z\right) \sin z-3 \cos z / z^{2}$. The dynamic mass density does not depend on the direction of the matrix acceleration. It is convenient to choose this direction along axis $z$. As for the case of cylindrical inclusions, the integral of $\vec{u} \cdot \vec{e}_{z}$ over the volume of the sphere must be different from zero for the components which contribute to the dynamic density. The computation of these integrals has been performed, leading to the result that the only contributions of the partial solutions correspond to $m=0, n=1$. All other components do not contribute to the dynamic mass density.

Finally, the dynamic displacement field is given by

$$
\begin{aligned}
& u_{r}=\frac{1}{r}\left[A U_{1}(\alpha r)+C U_{3}(\beta r)\right] \cos \theta, \\
& u_{\theta}=\frac{-1}{r}\left[A V_{1}(\alpha r)+C V_{3}(\beta r)\right][\sin \theta],
\end{aligned}
$$

with

$$
\begin{aligned}
& U_{1}(\alpha r)=j_{1}(\alpha r)-\alpha r j_{2}(\alpha r), \\
& U_{3}(\beta r)=2 j_{1}(\beta r), \\
& V_{1}(\alpha r)=j_{1}(\alpha r), \\
& V_{3}(\beta r)=2 j_{1}(\beta r)-\beta r j_{2}(\beta r) .
\end{aligned}
$$

It is noteworthy that the structure of the displacement field presents strong similarities with the transversal displacement field within the cylinder, but involves spherical Bessel functions instead of usual Bessel functions.

\section{B. Resonance frequencies}

The non-dimensional resonance frequencies are obtained again by the determinant of the homogeneous system corresponding to a null displacement at $r=a$. It leads to the equation in $\omega^{*}=\omega \cdot a / c_{s}$,

$$
\begin{aligned}
& k_{c} \omega^{*} j_{2}\left(k_{c} \omega^{*}\right) j_{2}\left(\omega^{*}\right)-j_{1}\left(k_{c} \omega^{*}\right) j_{2}\left(\omega^{*}\right) \\
& -2 k_{c} j_{1}\left(\omega^{*}\right) j_{2}\left(k_{c} \omega^{*}\right)=0 .
\end{aligned}
$$

Having solved this equation and obtained the eigenfrequencies $\omega^{p *}$, the boundary condition leads also to the ratio $q^{p}=C / A$ for each eigenfrequency, with

$$
\begin{aligned}
q^{p} & =\frac{C}{A}=-\frac{U_{1}\left(k_{c} \omega^{p *}\right)}{U_{3}\left(\omega^{p *}\right)}=-\frac{V_{1}\left(k_{c} \omega^{p *}\right)}{V_{3}\left(\omega^{p *}\right)} \\
& =\frac{k_{c} \omega^{p *} j_{2}\left(k_{c} \omega^{p *}\right)-j_{1}\left(k_{c} \omega^{p *}\right)}{2 j_{1}\left(\omega^{p *}\right)} .
\end{aligned}
$$

As for the case of cylindrical inclusions, it is useful to study the case of incompressibility when $k_{c}$ tends to 0 . In this case, the eigenfrequencies equation becomes $j_{2}\left(\omega^{*}\right)=0$, and the eigenfrequencies are the zeros of spherical Bessel function $j_{2}$.

\section{Participation factors}

The participation factors are computed from the integrals $I$ and $M$ with $I=\int \vec{u} \cdot \vec{e}_{z} d V$, and $M=\int\|\vec{u}\|^{2} d V$. These quantities are given by

$$
I=\frac{4 \pi A a^{2}}{3}\left[j_{1}\left(k_{c} \omega^{p *}\right)+2 q^{p} j_{1}\left(\omega^{p *}\right)\right]
$$

and

$$
\begin{aligned}
M= & \int\left\|\mathbf{u}_{p}\right\|^{2}=\frac{4 \pi a A^{2}}{3}\left\{Q_{11}\left(k_{c} \omega^{p *}\right)+4 q^{p} j_{1}\left(k_{c} \omega^{p *}\right)\right. \\
& \left.\times j_{1}\left(\omega^{p *}\right)+\left(q^{p}\right)^{2} Q_{33}\left(\omega^{p *}\right)\right\},
\end{aligned}
$$


where

$$
\begin{aligned}
& Q_{11}(x)=j_{1}^{2}-\frac{5}{2} x j_{1} j_{2}+\frac{x^{2}}{2}\left(j_{1}^{2}+j_{2}^{2}\right), \\
& Q_{33}(x)=4 j_{1}^{2}-5 x j_{1} j_{2}+x^{2}\left(j_{1}^{2}+j_{2}^{2}\right),
\end{aligned}
$$

$$
\begin{aligned}
k^{p} & =\frac{I^{2}}{V M} \\
& =\frac{\left[j_{1}\left(k_{c} \omega^{p *}\right)+2 q^{p} j_{1}\left(\omega^{p *}\right)\right]^{2}}{\left\{Q_{11}\left(k_{c} \omega^{p *}\right)+4 q^{p} j_{1}\left(k_{c} \omega^{p *}\right) j_{1}\left(\omega^{p *}\right)+q^{p 2} Q_{33}\left(\omega^{p *}\right)\right\}} .
\end{aligned}
$$

with $j_{1}=j_{1}(x), j_{2}=j_{2}(x)$.

As previously mentioned, the participation factors are finally given by introducing the averages $I / V, M / V$ over the volume $V=(4 / 3) \pi a^{3}$ of the sphere by
Introducing the values of the factors $q^{p}$ and taking into account the equation for eigenfrequencies leads finally to

$$
k^{p}=\frac{2 \alpha^{*} \beta^{*} j_{2}\left(\alpha^{*}\right) j_{2}\left(\beta^{*}\right)}{\alpha^{*} \beta^{*}\left(\beta^{*} j_{1}\left(\beta^{*}\right) j_{2}\left(\alpha^{*}\right)+\alpha^{*} j_{1}\left(\alpha^{*}\right) j_{2}\left(\beta^{*}\right)\right)-j_{1}\left(\alpha^{*}\right) j_{1}\left(\beta^{*}\right)\left(2 \alpha^{* 2}+\beta^{* 2}\right)} .
$$

When $k_{c}$ tends to zero, the participation factors tend also to 0 , because their numerators contain $j_{2}\left(\beta^{*}\right)=j_{2}\left(\omega^{p *}\right)$ which tends to 0 with $k_{c}$, as observed previously.

\section{Synthesis of the results for spherical inclusions}

The dynamic mass density [Eq. (2)] for spherical inclusions of radius $a$ is characterized by the eigenfrequencies that are solutions of

$$
k_{c} \omega^{*} j_{2}\left(k_{c} \omega^{*}\right) j_{2}\left(\omega^{*}\right)-j_{1}\left(k_{c} \omega^{*}\right) j_{2}\left(\omega^{*}\right)-2 k_{c} j_{1}\left(\omega^{*}\right) j_{2}\left(k_{c} \omega^{*}\right)=0,
$$

where $j_{1}, j_{2}$ are spherical Bessel functions.

The participation factors which enter the expression of the dynamic density through Eqs. (4) and (5) are given for each solution $\omega^{p *}$ of the previous equation by $K_{x x}^{p}=K_{y y}^{p}=K_{z z}^{p}=k^{p}$, where $k^{p}$ is given by

$$
k^{p}=\frac{2 k_{c} j_{2}\left(k_{c} \omega^{p *}\right) j_{2}\left(\omega^{p *}\right)}{k_{c} \omega^{p *}\left(j_{1}\left(\omega^{p *}\right) j_{2}\left(k_{c} \omega^{p *}\right)+k_{c} j_{1}\left(k_{c} \omega^{p *}\right) j_{2}\left(\omega^{p *}\right)\right)-j_{1}\left(k_{c} \omega^{p *}\right) j_{1}\left(\omega^{p *}\right)\left(1+2 k_{c}^{2}\right)} .
$$

\section{APPLICATIONS}

\section{A. Cylindrical inclusions}

As seen in Secs. III and IV, the resonance frequencies and participation factors depend only on the non-dimensional frequency $\omega^{*}=\omega \cdot a / c_{s}$. However, the coefficients appearing in the equation giving $\omega^{*}$ for the transversal motion depend on the ratio $k_{c}=c_{s} / c_{p}$, which itself depends only on Poisson's ratio $\nu$.

Figure 1 displays the non-dimensional resonance frequencies for the transversal motion related to the first six modes as a function of the Poisson's ratio. The part near $\nu$ $=0.5$ has been enhanced on the right part of the figure. The resonance frequencies increase moderately with $\nu$ for any mode, except when the Poisson's ratio is nearing $\nu=0.5$ where higher modes display a significant increase near this value of $\nu$. In all cases, the value of the eigenfrequency for $\nu=0.5$ has been computed by using the zeros of $J_{2}$, confirming the limit of the eigenfrequencies when $\nu$ tends to 0.5 . All curves display a plateau near $\nu=0.5$, which is very short for the sixth resonance frequency, but is clear on the enhanced figure at the right.
The participation factors related to these first six modes have been displayed in Fig. 2. The left part corresponds to all values of Poisson's ratios and the right part to $\nu$ between 0.45 and 0.5 . It shows that the first mode contains the main participation to the dynamic mass density with a participation factor being near to 0.7 for small values of the Poisson's ratio. However, when the Poisson's ratio increases, the first participation factor decreases, the highest participation factor being successively the one related to increasing ranks of modes, as show the values corresponding to $\nu$ nearing 0.5 .

All participation factors tend to 0 for $\nu$ reaching 0.5 , as expected from the expression of the participation factors. These results show therefore that the use of nearly incompressible materials leads to a prediction of the dynamic mass density which is very sensitive to the value of the Poisson's ratio.

\section{B. Spherical inclusions}

The non-dimensional eigenfrequencies for spherical inclusions are reported in Fig. 3 and show values of 

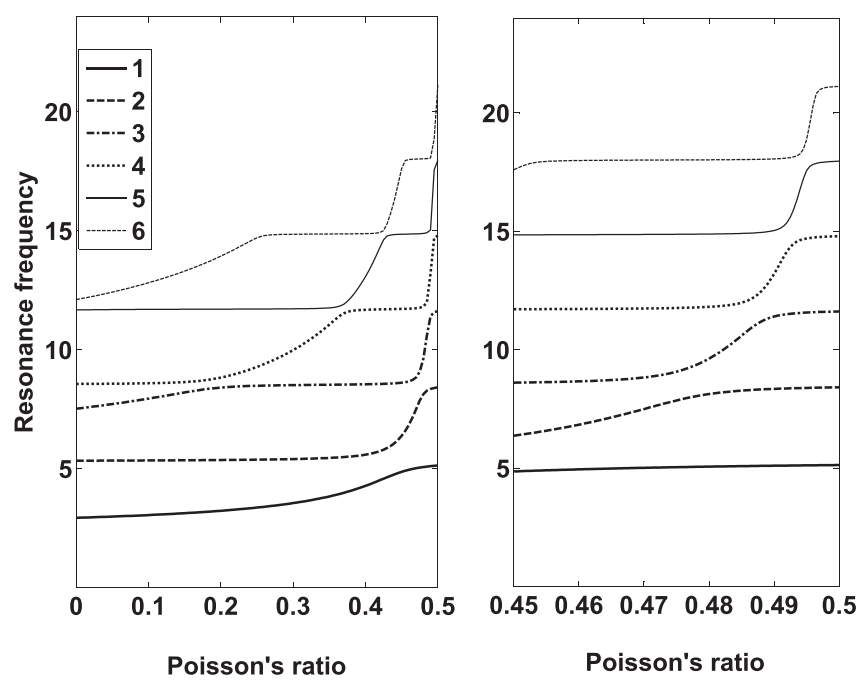

FIG. 1. Cylindrical inclusions. First six non-dimensional resonance frequencies for the transversal motion as functions of the Poisson's ratio. Right: elargement for Poisson's ratio between 0.45 and 0.5 .

eigenfrequencies that are higher than the ones for cylindrical inclusions with a trend being overall very similar to the one observed in the case of cylindrical inclusions.

The values of the related participation factors displayed in Fig. 4 are also very similar to the ones observed in the case of cylindrical inclusions, the value for the first mode being however lower than for cylindrical inclusions, at low values of $\nu$. The results also show a trend similar when the Poisson's ratio is nearing 0.5 , the highest participation factors being related to higher modes. It is noteworthy that the participation factors are closed form expressions of the eigenfrequencies. However, the eigenfrequencies are more difficult to obtain. So, in order to make easier a further use of this work, precise values of nondimensional eigenfrequencies have been reported in Appendix B.

An example of dynamic mass density has been reported in Fig. 5 for the case of a composite containing a matrix
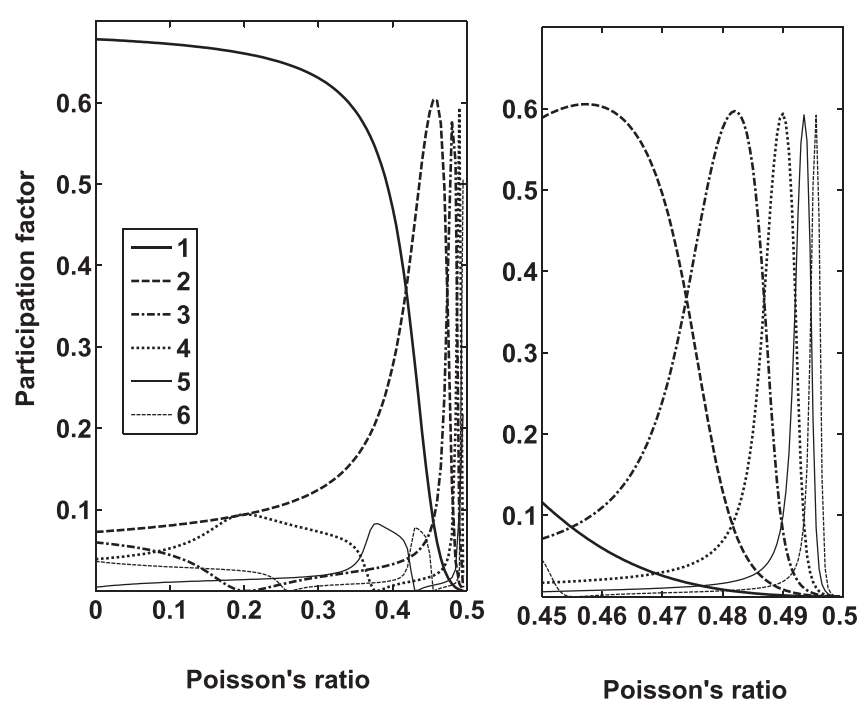

FIG. 2. Cylindrical inclusions. First six participation factors for the transversal motion as functions of the Poisson's ratio. Right: enlargement for Poisson's ratio between 0.45 and 0.5 .

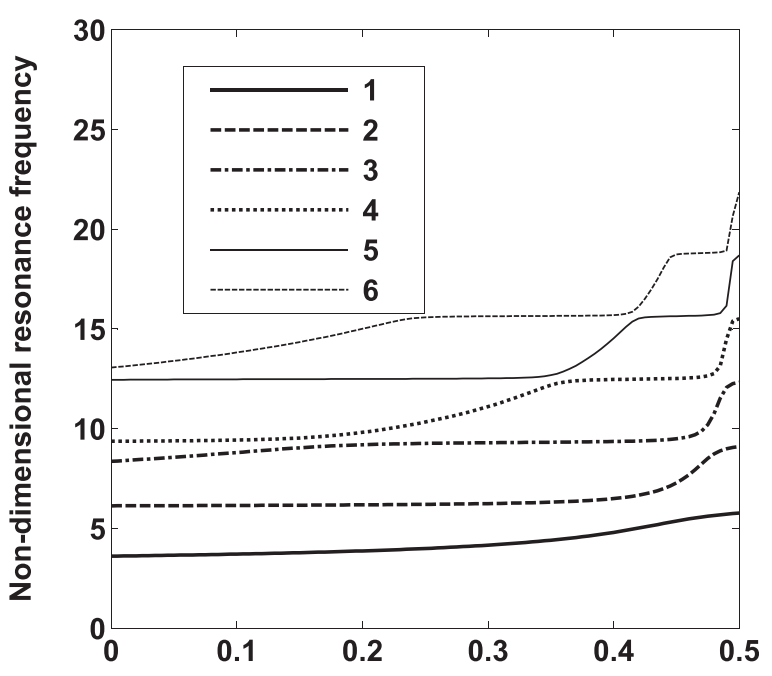

Poisson's ratio

FIG. 3. Spherical inclusions. First six non-dimensional resonance frequencies vs Poisson's ratio.

made of alumina (Young modulus $E_{a}=350 \mathrm{MPa}, \nu_{a}=0.25$, $\rho_{a}=3950 \mathrm{kgm}^{3}$ ) and spherical inclusions made of polystyrene $\left(E_{p}=2 \mathrm{MPa}, \nu_{p}=0.11, \rho_{p}=900 \mathrm{~kg} / \mathrm{m}^{3}\right)$ with a concentration of 0.5 and a radius of $2 \mathrm{~mm}$. The frequency range has been chosen to display the dynamic mass density $\rho^{\text {eff }}$ around the first two eigenfrequencies, showing the ranges where $\rho^{\text {eff }}$ is negative (highlighted by a bold line along the axis $\rho^{\text {eff }}=0$ ). The frequency range of practical interest where $\rho^{\text {eff }}$ is negative could be enlarged by using reinforced polystyrene containing inner inclusions with a material of higher density, which would increase significantly the density of the inclusion material and as a consequence the dynamic part of the density.

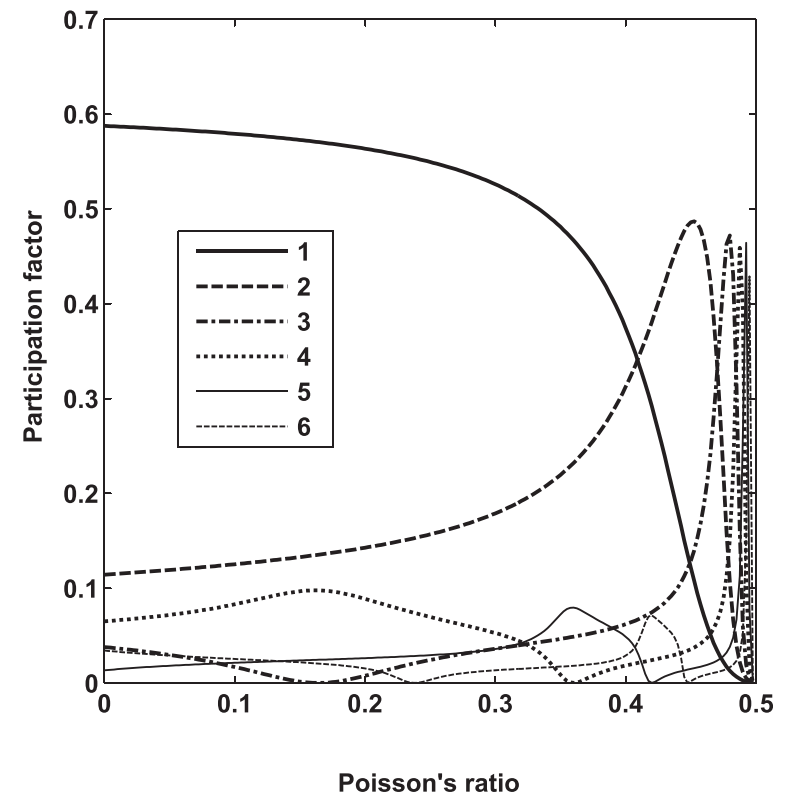

FIG. 4. Spherical inclusions. First six participation factors vs Poisson's ratio. 


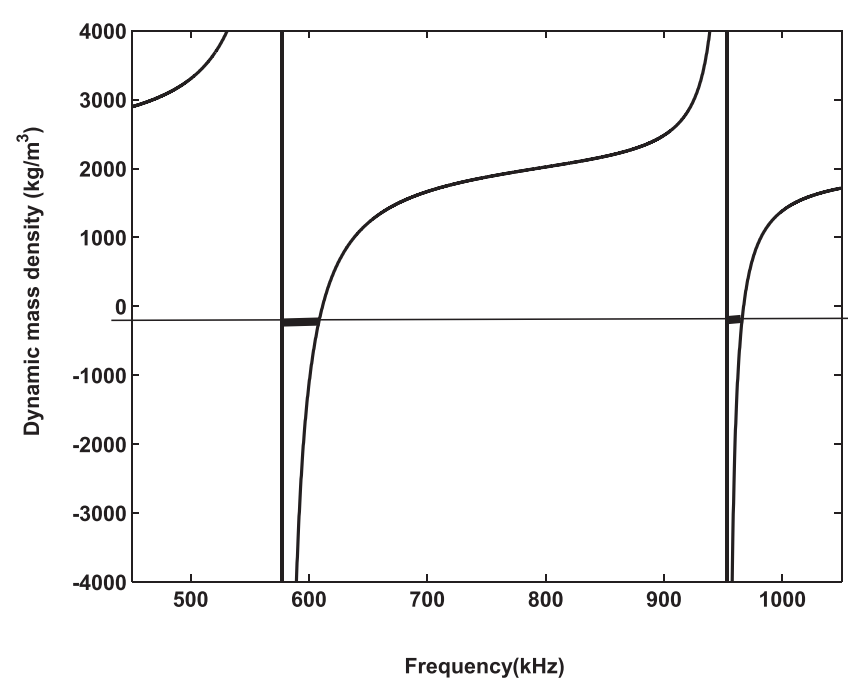

FIG. 5. Effective dynamic mass density vs frequency for a material matrix containing spherical inclusions of polystyrene.

\section{CONCLUSION}

In this paper, the basic results obtained from the homogenization theory using asymptotic expansions have been summarized for composite elastic metamaterials with homogeneous inclusions displaying inner resonance with negligible scattering. These results show, under specific conditions corresponding to very soft inclusions with a relatively high density, that the dynamic behaviour of the composite displays a frequency-dependent and tensorial dynamic mass density. This dynamic mass density can be computed knowing the resonance frequencies of the inclusions for the condition of null value at the surface of the inclusion of the relative displacement between matrix and inclusion. This greatly simplifies the computation of the dynamic mass density. In addition, it shows that all resonators are independent.

The resonance frequencies of these resonators have been computed successfully in the case of long cylindrical fibers or spherical inclusions. Having obtained these resonance frequencies, the participation factors related to the different modes are given by simple closed-form expressions. The results show that the participation factors decrease with increasing ranks of resonance frequencies at moderate values of the Poisson's ratio. In this case, the first modes constitute the main contribution to the dynamic mass density. When the material is nearly incompressible, the contribution of modes related to higher frequencies become more important and the dynamic mass density is very sensitive to the exact value of the Poisson's ratio of the inclusions.

It can be noticed that the dynamic mass density tends to infinity at the resonance frequencies, because the results have been obtained for a perfect elastic material. However, these results can be extended to viscoelastic materials in order to account for physical damping, leading to a finite dynamic mass density by using an asymptotic solution for a slight damping. ${ }^{28}$ Such an extension will be the subject of a further paper.

\section{APPENDIX A: THE CELL PROBLEM WITHIN THE INCLUSION}

The cell problem over the inclusion can be obtained ${ }^{25,26}$ by using a homogenization method based on double scale asymptotic expansion, ${ }^{27}$ whose main aspects are recalled thereafter.

The microscopic structure is assumed periodic with a periodic cell $\Omega$, split into $\Omega_{m}$ and $\Omega_{i}$ for matrix and inclusion, separated by the interface $\Gamma$ between matrix and inclusion. One considers that the ordering is such that $\Omega_{m}$ is connected over all the domain, while $\Omega_{i}$ is made of separate inclusion domains.

The double scale method can be described as follows: the position of a point at the macroscopic scale is determined by using the so-called "slow position vector" $\vec{x}$, while the position of a point at the microscopic scale (i.e., inside the periodic cell) is determined by the "fast position vector" $\vec{y}=\vec{x} / \epsilon$, leading to an amplification of the fluctuation of physical variables when looking at the microscopic scale. The components of the displacement field are assumed to be functions of fast and slow variables by $\vec{u}=\vec{u}(\vec{x}, \vec{y})$ and their dependence in $\vec{y}$ is periodic. Elasticity parameters and densities are also periodic functions of $\vec{y}$, by construction of the composite material.

The displacement field is searched by using an asymptotic expansion of the form $\vec{u}(\vec{x}, \vec{y})=\vec{u}_{(0)}(\vec{x}, \vec{y})$ $+\epsilon \vec{u}_{(1)}(\vec{x}, \vec{y})+\cdots$. Finally, the spatial derivatives $\partial / \partial x_{j}$ within local dynamic equations are replaced by "double scale spatial derivatives" $\partial / \partial x_{j}+(1 / \epsilon)\left(\partial / \partial y_{j}\right)$. The factor in front of the second derivative accounts for the enhancement of the fluctuations at the local scale.

The asymptotic expansion of the displacement field can be introduced into the dynamic equations (within matrix and inclusion) and continuity equations of displacement and traction over $\Gamma$. Using the double scale derivatives, it produces a set of cell problems that can be solved sequentially to obtain the displacement fields over matrix and inclusion.

The displacement field within the matrix is found to $\mathrm{be}^{25,26} \vec{u}^{(m)}(\vec{x}, \vec{y})=\vec{U}^{(m)}(\vec{x})+\epsilon \vec{u}_{(1)}^{(m)}(\vec{x}, \vec{y})$, where $\vec{u}_{(1)}^{(m)}$ is the solution of the cell problem corresponding to the static homogenization within the matrix for a macroscopic strain tensor computed from $\vec{U}^{(m)}(\vec{x})$, with null traction over $\Gamma$. Indeed, at this stage, due to the large wavelength within the matrix, the inertia term does not contribute to the localization problem within the matrix.

The first order components $u_{j(0)}^{(i)}$ of the displacement field $\vec{u}_{(0)}^{(i)}$ within the inclusions are then obtained by solving the first order cell problem for $\vec{u}_{(0)}^{(i)}$,

$$
\begin{aligned}
& \frac{\partial}{\partial y_{k}}\left(\lambda^{(i)} \varepsilon_{l l(0)(y)}^{(i)} \delta_{j k}+\mu^{(l)} \varepsilon_{i k(0)(y)}^{(i)}\right)+\rho^{(l)} \omega^{2} u_{j(0)}^{(i)}=0, \\
& u_{j(0)}^{(i)}(\vec{x}, \vec{y})=\vec{U}^{(m)}(\vec{x}), \quad \vec{y} \in \Gamma,
\end{aligned}
$$

where $\varepsilon_{i k(0)(y)}^{(i)}$ stands for the components of the strain tensor within the inclusion computed from $\vec{u}_{(0)}^{(i)}$ by using $y$ derivatives.

Equation (3) is then obtained by making the change of variable $\vec{w}=\vec{u}_{(0)}^{(i)}-\vec{U}^{(m)}(\vec{x})$. 


\section{APPENDIX B: DETAILED VALUES OF EIGENFREQUENCIES}

Values of non-dimensional eigenfrequencies for transversal motion of cylindrical inclusions.

\begin{tabular}{lcccccccc}
\hline \hline Frequency rank $\rightarrow$ & 1 & 2 & 3 & 4 & 5 & 6 & 7 & 8 \\
\hline$\downarrow$ Poisson's ratio & & & & & & & & \\
0 & 2.9345 & 5.334 & 7.519 & 8.561 & 11.670 & 12.106 & 14.861 & 16.554 \\
0.1 & 3.050 & 5.346 & 7.944 & 8.590 & 11.694 & 12.812 & 14.865 & 17.545 \\
0.2 & 3.230 & 5.364 & 8.408 & 8.828 & 11.704 & 13.930 & 14.876 & 18.009 \\
0.3 & 3.553 & 5.403 & 8.511 & 9.981 & 11.718 & 14.851 & 15.977 & 18.019 \\
0.4 & 4.277 & 5.588 & 8.548 & 11.678 & 13.069 & 14.877 & 18.013 & 20.865 \\
0.5 & 5.136 & 8.417 & 11.620 & 14.796 & 17.960 & 21.117 & 24.270 & 27.421 \\
\hline \hline
\end{tabular}

Values of non-dimensional eigenfrequencies for spherical inclusions.

\begin{tabular}{lcccccccc}
\hline \hline Frequency rank $\rightarrow$ & 1 & 2 & 3 & 4 & 5 & 6 & 7 & 8 \\
\hline$\downarrow$ Poisson's ratio & & & & & & & & \\
0 & 3.607 & 6.129 & 8.357 & 9.365 & 12.444 & 13.057 & 15.642 & 17.538 \\
0.1 & 3.709 & 6.148 & 8.800 & 9.425 & 12.472 & 13.815 & 15.650 & 18.553 \\
0.2 & 3.870 & 6.178 & 9.193 & 9.813 & 12.488 & 15.000 & 15.678 & 18.789 \\
0.3 & 4.157 & 6.241 & 9.291 & 11.101 & 12.518 & 15.632 & 17.223 & 18.807 \\
0.4 & 4.798 & 6.494 & 9.354 & 12.461 & 14.521 & 15.690 & 18.797 & 21.908 \\
0.5 & 5.763 & 9.095 & 12.323 & 15.515 & 18.689 & 21.854 & 25.013 & 28.168 \\
\hline \hline
\end{tabular}

${ }^{1}$ E. Shamonina and L. Solymar, "Metamaterials: How the subject started," Metamaterials 1,12-18 (2007).

${ }^{2}$ V. G. Veselago, "The electrodynamics of substances with simultaneously negative values of $\varepsilon$ and $\mu$," Sov. Phys. Usp. 10, 509-514 (1968).

${ }^{3}$ J. O. Vasseur, P. A. Deymier, G. Prantziskonis, and G. Hong, "Experimental evidence for the existence of absolute acoustic band gaps in two-dimensional periodic composite media," J. Phys.: Condens. Mater. 10, 6051-6064 (1998).

${ }^{4}$ Z. Liu, X. Zhang, Y. Mao, Y. Y. Zhu, Z. Yang, C. T. Chan, and P. Sheng, "Locally resonant materials," Science 289, 1734-1736 (2000).

${ }^{5} \mathrm{~A}$. Baz, "An active acoustic metamaterial with tunable effective density," ASME J. Vib. Acoust. 132(4), 041011 (2010).

${ }^{6} \mathrm{~N}$. Cselyuszka, M. Secujski, and V. Crnojevic-Bengin, "Novel negative mass density resonant metamaterial unit cell," Phys. Lett. A 379, 33-36 (2015).

${ }^{7}$ H. H. Huang and C. T. Sun, "Locally resonant acoustic metamaterials with 2D anisotropic effective mass density," Philos. Mag. 91(6), 981-996 (2011).

${ }^{8} \mathrm{H}$. H. Huang and C. T. Sun, "Anomalous wave propagation in a onedimensional acoustic metamaterial having simultaneously negative mass density and Young's modulus," J. Acoust. Soc. Am. 132(4), 2887-2895 (2012).

${ }^{9}$ S. H. Lee, C. M. Park, Y. M. Seo, Z. G. Wang, and C. K. Kim, "Acoustic metamaterial with negative density," Phys. Lett. A. 373(48), 4464-4469 (2009).

${ }^{10}$ C. J. Naify, C. M. Chang, G. McKnight, and S. R. Nutt, "Scaling of membrane-type locally resonant acoustic metamaterial arrays," J. Acoust. Soc. Am. 132(4), 2784-2792 (2012).

${ }^{11}$ J. Park, B. Park, D. Kim, and J. Park, "Determination of effective mass density and modulus for resonant metamaterials," J. Acoust. Soc. Am. 132(4), 2793-2799 (2012).

${ }^{12}$ P. Sheng, X. X. Zhang, Z. Liu, and C. T. Chan, "Locally resonant sonic materials," Physica B: Condensed Matter. 338, 201-205 (2003).

${ }^{13}$ Z. Yang, J. Mei, M. Yang, N. H. Chan, and P. Sheng, "Membrane-type acoustic metamaterial with negative dynamic mass," Phys. Rev. Lett. 101, 204301 (2008).

${ }^{14}$ S. Yao, X. Zhou, and G. Hu, "Experimental study on negative effective mass in a 1D mass-spring system," New J. Phys. 10, 043020 (2008).

${ }^{15} \mathrm{G}$. Ma and P. Sheng, "Acoustic metamaterials: From local resonances to broad horizons," Sci. Adv. 2, e1501595 (2007).
${ }^{16}$ Y. Ding, Z. Liu, C. Qiu, and J. Shi, "Metamaterial with simultaneously negative bulk modulus and mass density," Phys. Rev. Lett. 99, 093904 (2010).

${ }^{17}$ R. Gracia-Salgado, D. Torrent, and J. Sanchez-Dehesa, "Double-negative acoustic metamaterials based on quasi-two-dimensional fluid-like shells," New J. Phys. 14, 103052 (2012).

${ }^{18} \mathrm{Z}$. Liu, C. T. Chan, and P. Sheng, "Analytic model of phononic crystals with local resonances," Phys. Rev. B 71, 014103 (2005).

${ }^{19}$ J. Mei, Z. Liu, W. Wen, and P. Sheng, "Effective mass density of composites," Phys. Rev. B 76, 134205 (2007).

${ }^{20}$ Y. Wu, Y. Lai, and Z.-Q. Zhang, "Effective medium theory for elastic metamaterials in two dimensions," Phys. Rev. B 76, 205313 (2007).

${ }^{21}$ M. Yang, G. Ma, Y. Wu, Z. Yang, and P. Sheng, "Homogenization scheme for acoustic metamaterials," Phys. Rev. B 89, 064309 (2014).

${ }^{22}$ B. Yuan, V. F. Humphrey, J. Wena, and X. Wena, "On the coupling of resonance and Bragg scattering effects in three-dimensional locally resonant sonic materials," Ultrasonics 53, 1332-1343 (2013).

${ }^{23} \mathrm{X}$. Zhou and G. Hu, "Analytic model of elastic metamaterials with local resonances,” Phys. Rev. B 79, 195109 (2009).

${ }^{24} \mathrm{P}$. Sheng, Introduction to Wave Scattering, Localization and Mesoscopic Phenomena (Academic Press, New York, 1995), p. 66.

${ }^{25}$ J. L. Auriault and G. Bonnet, "Dynamique des composites élastiques périodiques" ("Dynamics of elastic periodic composites"), Arch. Mech. 37(4), 269-284 (1985).

${ }^{26}$ J. L. Auriault, "Acoustics of heterogeneous media: Macroscopic behavior by homogenization," Current Topics Acoust. Res. 1, 63-90 (1994).

${ }^{27}$ A. Bensoussan, J. L. Lions, and G. Papanicolaou, Asymptotic Analysis for Periodic Structures (North-Holland, Amsterdam, 1978), pp. 1-392.

${ }^{28}$ J. L. Auriault and C. Boutin, "Long wavelength inner-resonance cut-off frequencies in elastic composite materials," Int. J. Solids Struct. 49(23-24), 3269-3281 (2012).

${ }^{29}$ A. Avila, G. Griso, and B. Miara, "Bandes phononiques interdites en élasticité linéarisée" ("Phononic bandgaps in linearized elasticity"), C. R. Acad. Sci. I 340(12), 933-938 (2005).

${ }^{30}$ N. O. Babych, I. V. Kamotski, and V. P. Smyshlyaev, "Homogenization of spectral problems in bounded domains with doubly high contrasts," Networks Heterogeneous Media 3(3), 413-436 (2008).

${ }^{31}$ N. S. Bakhvalov and G. P. Panasenko, Homogenization Averaging Process in Periodic Media. Mathematical Problems in the Mechanics of Composite Materials (Kluwer, Dordrecht, 1989), pp. 1-366.

${ }^{32}$ J. Carbonell, F. Cervera, J. Sanchez-Dehesa, J. Ariaga, L. Gumen, and A. Krokhin, "Homogenization of two-dimensional anisotropic dissipative photonic crystal," Appl. Phys. Lett. 97(4), 231122 (2010).

${ }^{33} \mathrm{G}$. W. Milton, "New metamaterials with macroscopic behavior outside that of continuum elastodynamics," New J. Phys. 9, 359 (2007).

${ }^{34} \mathrm{G}$. W. Milton and J. R. Willis, "On modifications of Newton's second law and linear continuum elastodynamics," Proc. R. Soc. A. 463, 855-880 (2007).

${ }^{35}$ S. Nemat-Nasser, J. R. Willis, A. Srivastava, and A. V. Amirkhizi, "Homogenization of periodic elastic composites and locally resonant materials," Phys. Rev. B 83, 104103 (2011).

${ }^{36}$ A. N. Norris, A. L. Shuvalov, and A. A. Kutsenko, "Analytical formulation of three-dimensional dynamic homogenization,” Proc. R. Soc. A 468, 1629-1651 (2012).

${ }^{37}$ W. J. Parnell and I. D. Abrahams, "Dynamic homogenization in periodic fibre reinforced media. Quasi-static limit for SH waves," Wave Motion 43, 474-498 (2006).

${ }^{38}$ J. Soubestre and C. Boutin, "Non-local dynamic behavior of linear fiber reinforced materials," Mech. Mat. 55, 16-32 (2012).

${ }^{39}$ T. T. Dang, "Méthodes numériques pour l'homogénéisation élastodynamique des matériaux hétérogènes périodiques" ("Numerical methods for obtaining the dynamic effective properties of periodical heterogeneous materials"), Ph.D. thesis Université Paris-Est (2015).

${ }^{40}$ H. Nassar, Q.-C. He, and N. Auffray, "A generalized theory of elastodynamic homogenization for periodic media," Int. J. Solids Struct. 84, 139-146 (2016).

${ }^{41}$ H. Nassar, Q.-C. He, and N. Auffray, "On asymptotic elastodynamic homogenization approaches for periodic media,” J. Mech. Phys. Solids 88, 274-290 (2016).

${ }^{42}$ J. R. Willis, "Effective constitutive relation for waves in composites and metamaterials," Proc. R. Soc. A 467, 1865-1879 (2011). 
${ }^{43}$ G. Bonnet and V. Monchiet, "Low frequency locally resonant metamaterials containing composite inclusions," J. Acoust. Soc. Am. 137(6), 3263-3271 (2015).

${ }^{44}$ A. C. Eringen and E. S. Suhubi, Elastodynamics, Vol. 2 (Academic Press, New York, 1975), pp. 772-776 and 806-809.

${ }^{45}$ A. E. H. Love, A Treatise on the Mathematical Theory of Elasticity (Dover Publications, New York, 1944), pp. 249-251.
${ }^{46}$ P. Debye, “Theorie der spezifischen Wärmen,” Ann. Phys. 344, 789-839 (1912).

${ }^{47}$ P. J. Schafbuch and F. J. Rizzo, "Eigenfrequencies of an elastic sphere with fixed boundary conditions," J. Appl. Mech. 59, 458-459 (1992).

${ }^{48}$ P. M. Morse and H. Feshbach, Methods of Theoretical Physics (McGrawHill, New York, 1953), p. 1264. 